\title{
Estimativa de matriz origem/destino utilizando dados do sistema de bilhetagem eletrônica: proposta metodológica
}

\author{
André Leite Guerra¹, Heloisa Maria Barbosa², Leise Kelli de Oliveira ${ }^{3}$
}

\begin{abstract}
Resumo: A caracterização da demanda é fundamental no desenvolvimento de redes de transporte, existindo inúmeros métodos para a análise da demanda, sendo que as metodologias convencionais apresentam custos e tempo de execução elevados, que restringem a frequência de sua aplicação. Visando mitigar as deficiências dos métodos convencionais de pesquisas de transporte, este artigo apresenta uma proposta metodológica de estimativa de uma Matriz Origem-Destino para sistemas de transporte público por ônibus usando informações do Sistema de Bilhetagem Eletrônica e do banco de dados das linhas em Sistema de Informação Geográfica (SIG). Tal proposta consiste na montagem da rede de transporte em SIG, na caracterização e tratamento dos dados de bilhetagem, na determinação da matriz semente e nos procedimentos para a estimativa da matriz final. A metodologia foi testada para o sistema de transporte municipal de Maceió/AL. Os resultados obtidos mostraram-se consistentes e indicam que a metodologia permite incorporar as mudanças ocorridas no sistema de transporte. Além disso, a aplicação da metodologia indicou que esta é simples, conveniente e prática, podendo ser utilizada para estudos relacionados a transporte público.
\end{abstract}

Palavras-chave: Matriz O/D. Bilhetagem eletrônica. Transporte público.

\begin{abstract}
The characterization of demand is fundamental in the development of transport networks. There are numerous methods for the demand analysis, and the conventional methods have high costs and time of implementation, that restrict the frequency of its application. In order to mitigate the weaknesses of conventional methods of transport surveys, this paper presents a methodology for estimation of an Origin-Destination Matrix for public bus transport systems using information from the Automatic Fare Collection and from Geographic Information Systems (GIS) database of buses lines. This methodology consists in create the transport network in GIS, the characterization and processing of fare data for determining the seed matrix and procedures to estimate the final matrix. The methodology was tested for the municipal transportation system of Maceió/AL. The results were consistent and indicate that the proposed methodology allows incorporating the changes in the transport system. Moreover, the application of the methodology indicated its simplicity, convenience and practicality, and can be used for studies related to public transportation.
\end{abstract}

Keywords: OD Matrix. Automatic Fare Collection. Public transportation.

\section{INTRODUÇÃO}

O planejamento urbano é uma atividade fundamental para que se consiga ordenar e encaminhar o desenvolvimento das cidades. A ausência deste planejamento certamente explica parte dos problemas observados na maioria dos municípios brasileiros. Planejar o espaço urbano, tanto a sua estrutura física como os seus aspectos de uso e ocupação, significa, muitas vezes, focar em programas relacionados ao sistema de transporte e sua infraestrutura, visto ser este um dos principais ordenadores do espaço. As relações entre a ocupação e o uso do solo com a infraestrutura de transporte explicam e condicionam o desenvolvimento das cidades (ANTP,

\footnotetext{
1 Universidade Federal de Minas Gerais - Mestrado em Geotecnia e Transportes (andreguerra@gmail.com).

2 Universidade Federal de Minas Gerais - Mestrado em Geotecnia e Transportes (helobarb@etg.ufmg.br).

3 Universidade Federal de Minas Gerais - Mestrado em Geotecnia e Transportes (leise@etg.ufmg.br).
}

Manuscrito recebido em 14/05/2014 e aprovado para publicação em 08/08/2014. Este artigo é parte de TRANSPORTES v. 22, n. 3, 2014. ISSN: 2237-1346 (online).

DOI: http://dx.doi.org/10.14295/transportes.v22i3.789.
1997). Neste cenário, a realização apropriada do planejamento necessita de uma melhor compreensão das relações de uso do solo e de sua integração com o sistema de transporte.

Por outro lado, o transporte é responsável por uma demanda existente ou futura e o dimensionamento da oferta é uma tarefa fundamental no equacionamento e desenvolvimento da rede de transporte. Neste contexto, é necessário qualificar e categorizar a demanda (espacial e temporal) para estabelecer os padrões de viagem, o comportamento dos fluxos, as possibilidades de atendimento em função da estrutura viária, dentre outros elementos. Entretanto, o ambiente urbano é extremamente dinâmico e mutável dificultando, muitas vezes, o reconhecimento desses padrões.

$\mathrm{Na}$ busca de informações necessárias ao desenvolvimento das soluções, surgiram diversos modelos de demanda na tentativa de auxiliar o planejamento de transporte. Dentre estes, merecem atenção aqueles que procuram captar os desejos de viagem dos habitantes, coletando não só a origem e o destino de seus deslocamentos 
como também as variáveis de tempo a ele associados (início, fim, duração), o modo de transporte utilizado e os motivos da realização do deslocamento, além de informações socioeconômicas, consubstanciando as chamadas Matrizes de Origem-Destino (Matrizes OD).

Para a estimativa dessas matrizes podem ser utilizados métodos indiretos, através de modelos matemáticos de previsão de demanda, ou métodos diretos, que se baseiam na realização de pesquisa de campo, sendo esta a mais utilizada. Embora seja uma prática relativamente comum, verifica-se que os empecilhos de realização são cada vez maiores devido às dificuldades de acesso aos entrevistados em função de problemas de segurança. Além disso, os custos e o tempo de execução deste tipo de pesquisa são muito expressivos, restringindo a frequência de sua aplicação para prazos mais alongados.

Em paralelo, atualmente estão disponíveis várias tecnologias que, quando utilizadas nos veículos ou de posse pessoal dos usuários, poderiam ser aproveitadas para coleta de dados de transporte. Estas tecnologias vêm sendo adotadas pelos sistemas de transporte para fins específicos, gerando um excelente conjunto de informações, geralmente não utilizadas para aprimorar os estudos na área. Dentre estes, merece destaque a bilhetagem eletrônica, um sistema de cobrança de tarifa que armazena informações de todas as transações efetuadas no transporte público através do uso de cartões eletrônicos individuais.

Neste contexto, considerando ampliar o uso de informações disponíveis para o aprimoramento das atividades de planejamento e programação dos sistemas de transportes, este artigo apresenta uma proposta metodológica para a estimativa de uma matriz origem-destino específica para o transporte público por ônibus. Esta proposta se justifica devido aos elevados custos financeiros e administrativos que envolvem a realização de uma pesquisa Origem-Destino (OD), nas atividades em campo e consolidação de dados em escritório, que faz com que a sua periodicidade seja naturalmente baixa e a sua amostra relativamente pequena (cerca de 5\% do universo). De modo geral, a pesquisa é realizada a cada dez anos e, este período de tempo acarreta novos problemas visto que o espaço urbano está em constante processo de transformação e as informações obtidas em tram em obsolescência.
Assim, a busca de métodos alternativos para obtenção de matrizes OD que não dependam de levantamento de campo e sejam menos onerosas, é uma constante no meio técnico-acadêmico. Entre as alternativas, tratando-se apenas das viagens realizadas por transporte público coletivo, as que utilizam os dados de Sistemas de Bilhetagem Eletrônica (SBE) são as que parecem oferecer melhores perspectivas.

Para apresentar a metodologia proposta e os resultados da aplicação, este trabalho está assim dividido: na seção 2 é apresentado o referencial teórico do trabalho, abordando sistemas de coleta automática de dados, estudos realizados para a determinação de matriz origem destino utilizando tais sistemas e uma alternativa aos métodos tradicionais para estimar uma Matriz OD utilizando contagem volumétrica de fluxos em um conjunto de segmentos de rede. Na seção 3 é apresentada a proposta metodológica que consiste na montagem da rede de transporte em SIG, na caracterização e tratamento dos dados de bilhetagem, na determinação da matriz semente e nos procedimentos para a estimativa da matriz final. Os resultados da aplicação da metodologia estão apresentados na seção 4. As considerações finais encerram este artigo.

\section{PLANEJAMENTO DE TRANSPORTE UTILIZANDO COLETA AUTOMÁTICA DE DADOS}

Os sistemas de Coleta Automática de Dados (ADC) têm seu uso cada vez mais difundido em todo o mundo. Estes podem incluir os sistemas de Localização Automática de Veículo (AVL), de Contagem Automática de Passageiros (APC) e os Sistemas de Bilhetagem Eletrônica (SBE). Os sistemas de bilhetagem eletrônica, foco deste artigo, incluem várias tecnologias, sendo, o smart card, a opção mais moderna e flexível de pagamento de tarifa.

Segundo Trépanier et al. (2008), o uso desses cartões no transporte coletivo começou a ser explorado nos anos 2000 apesar de eles terem sido inventados no final da década de 1960. Gordillo (2006) destaca que esses cartões estão rapidamente se tornando o padrão global de tarifação nos sistemas de transporte público. Nas grandes cidades brasileiras, segundo o relatório anual do vale-transporte de 2012 produzido pela NTU (Associação Nacional das Empresas de Transportes Urbanos), os smart cards represen- 
tam $97 \%$ dos vales-transportes. Em 2000, o material mais utilizado era o papel impresso, representando $67 \%$ do total.

\subsection{Determinação de Matriz O/D utilizando Sistemas de Coleta Automática de Dados}

Segundo Cui (2006), o aumento da utilização dos Sistemas de Coleta Automática de Dados (ADC) nos sistemas de transporte público em todo o mundo, implicou no desenvolvimento de pesquisas envolvendo estimativa de matrizes OD a partir dos dados gerados por estes sistemas de coleta. Uma vez que os órgãos gestores dispõem de diferentes informações, as pesquisas tendem a ter uma diferença tanto no foco como na metodologia. As principais vantagens destes métodos apontadas pelo referido autor são:

Redução de Custo: Além de não envolver equipes de pesquisa, os equipamentos são adquiridos previamente para outros propósitos e são projetados para coletar e arquivar as transações dos passageiros e os eventos do veículo em todas as viagens equipadas;

Elevada amostra: O tamanho da amostra do método baseado em entrevistas é sempre limitado devido aos altos custos envolvidos (geralmente menor que $5 \%$ do universo);

Maior frequência de execução: $O$ custo de desenvolver um sistema automatizado que processa as informações produzidas pelos ADC e gera a Matriz $\mathrm{O} / \mathrm{D}$, acontecerá apenas na primeira vez. Em todas as outras vezes, basta coletar informações mais recentes;

Aponta a necessidade de pesquisas por entrevistas mais específicas: Quando os resultados da matriz $\mathrm{O} / \mathrm{D}$, feita pelo método que utiliza $\mathrm{ADC}$, identificam certas áreas de especial interesse, uma pesquisa por entrevista mais direcionada pode ser conduzida para se obter informações mais detalhadas, não disponíveis nos sistemas ADC.

As metodologias já desenvolvidas para os sistemas sobre trilhos e para os sistemas por ônibus possuem uma diferença básica já que no primeiro tipo de sistema o pagamento da tarifa ocorre nas estações, enquanto no segundo, quase sempre, o pagamento é feito no interior do veículo. Quando o pagamento é feito nas estações, a origem do passageiro é determinada de forma direta como sendo a estação onde ocorreu a transação. Já nos sistemas por ônibus, os dados gerados pelos AVL devem ser utilizados de forma complementar. Assim, a origem do passageiro é referenciada pela posição geográfica do veículo no momento em que o passageiro efetuou a validação do seu cartão.

Outro fator que diferencia as metodologias em sua essência é que, em alguns casos, o controle de acesso é feito tanto na entrada como na saída, sendo que as estimativas de origem e destino são feitas de maneira bastante precisa. Segundo Zhao (2004), quando há apenas o controle de entrada, algumas suposições devem ser feitas para se inferir o local de desembarque do passageiro, tais como (i) o passageiro inicia sua próxima viagem no destino da viagem anterior; e (ii) o passageiro termina a sua última viagem do dia onde ele iniciou a primeira.

Zhao (2004) desenvolveu uma metodologia para a inferência do destino de um passageiro em um sistema de trilhos para Chicago Transit Authority, associando dados do SBE com dados do AVL e utilizando uma rede em SIG. Neste trabalho é avaliada a integração trilho-ônibus utilizando as informações de transferência para o ônibus para melhorar a matriz de passageiros do transporte sobre trilhos. Chicago Transit Authority continuou a evoluir seus estudos e, dois anos depois, Cui (2006) publicou uma metodologia para estimar uma Matriz O/D para ônibus a partir de dados obtidos de múltiplos sistemas ADC. Sua tese foca no sistema com controle apenas de entrada.

Trépanier et al. (2007 apud Chapleau et al., 2008; Munizaga et al., 2010) propuseram um método para estimar o ponto de desembarque de uma viagem em sistemas com controle de acesso apenas na entrada. A aplicação foi feita na cidade de Gatineau (Canadá). Posteriormente, Trépanier et al. (2008) desenvolveram uma metodologia para comparar os dados provenientes do SBE com os provenientes da pesquisa OD. Embora não se tenha obtido boas conclusões, os autores reforçam que a publicação é uma primeira comparação entre dados obtidos da bilhetagem eletrônica e dados obtidos de uma pesquisa OD.

Utilizando dados do SBE da cidade de Chang Chu (China), Lianfu et al. (2007) propuseram um método para construir uma matriz OD com nível de precisão no ponto de parada. O método não utiliza dados do sistema AVL e a referência com os pontos de parada é feita através das informações obtidas por meio de um questionário preenchido a bordo pelo motorista com 
os horários de cada parada.

No Brasil, Farzin (2008) aplicou sua metodologia na cidade de São Paulo, utilizando três fontes primárias de dados para obter a matriz: as transmissões do sistema AVL, a localização geográfica dos pontos de parada das linhas em análise e a base de dados do SBE. Neste trabalho, os destinos são inferidos conforme metodologia proposta por Zhao (2004) e não é contemplada a etapa de expansão da matriz para estimar todas as viagens do sistema, sendo considerados apenas os passageiros que embarcaram em veículos equipados com GPS (em 2006, o sistema AVL não estava presente em toda a frota). Os resultados obtidos através da metodologia proposta, utilizando dados de 2006, são comparados com a matriz OD realizada na cidade no ano de 1997. Apesar da semelhança nos resultados, algumas diferenças surgiram em função de fatores como: (i) a ocorrência de transformações na cidade durante o período entre 1997 e 2006; (ii) a matriz de 2006 captura apenas os padrões de viagem dos usuários de cartão eletrônico; (iii) a matriz obtida contempla apenas os passageiros que embarcam em veículos equipados com AVL.

Um estudo mais recente foi desenvolvido em Santiago, no Chile, por Munizaga et al. (2010). Na metodologia, o ponto de desembarque (destino) foi tomado como sendo o que minimizasse a distância de caminhada entre ele e o ponto de embarque seguinte. No caso do embarque nas estações de ônibus, em que não é identificada a linha utilizada, foi aplicado o conceito de "linhas comuns de ônibus", que se baseia na frequência das linhas para estimar aquela em que o passageiro embarcou. A metodologia proposta considerou a conectividade entre as linhas. Assim, na aplicação, foi possível determinar o destino de $82 \%$ das viagens. Dentre os $18 \%$ restantes, houve casos em que não existia ligação entre a linha embarcada e a linha seguinte, considerando-se uma distância aceitável de caminhada.

Em continuidade ao trabalho anterior, $\mathrm{Mu}-$ nizaga e Palma (2012) publicaram um trabalho acrescentando a etapa de expansão da amostragem. Foi utilizado um fator de expansão diferente para cada um dos três casos: quando a origem é conhecida, mas o destino não pode ser estimado; quando nem a origem, nem o destino puderam ser estimados; viagens não detectadas pelos cartões (caso de evasão de tarifa). Os autores propuseram uma solução simples, mas re- comendam que seja estudada uma solução mais sofisticada, principalmente no que se refere aos casos de evasão em que são requeridas informações adicionais.

\subsection{Determinação de Matriz OD Através de Contagens de Fluxo}

Uma corrente alternativa aos métodos tradicionais busca estimar uma Matriz OD utilizando contagem volumétrica de tráfego em um conjunto de segmentos da rede. Tal abordagem foi inicialmente proposta na década de 1970 para apoiar métodos expeditos de análise da demanda - em geral de curto prazo, já que não permite correlacionar a demanda com seus fatores causais (Willumsen, 1981). Para Bertoncini (2007), este processo de obtenção da demanda pode ser considerado como o inverso dos métodos tradicionais. Nele, a Matriz OD é estimada a partir dos fluxos em trechos da rede sendo que nos métodos tradicionais os fluxos são determinados a partir do conhecimento da matriz. Conforme exposto por Cascetta (1984), neste tipo de modelagem é estimada a matriz OD que minimiza uma medida de distância, "entrópica" ou euclidiana, em relação a uma matriz semente respeitando a restrição de que, uma vez alocada na rede, os volumes observados são reproduzidos nos arcos. Segundo Abrahamsson (1998), a matriz semente pode ser obtida de uma pesquisa amostral ou de matriz antiga (provavelmente desatualizada). Sendo assim, este tipo de modelo pode ser utilizado de forma complementar a outros métodos. As matrizes calculadas por estes modelos são denominadas Matrizes OD sintéticas.

Embora os métodos que envolvam a contagem de fluxos sejam abordados na literatura desde a década de 1970, eles não são amplamente conhecidos. Para Bertoncini et al. (2013), uma das razões disso deve-se ao fato de que existem questões de pesquisa relativas tanto à formulação do problema, quanto à sua solução, que ainda não foram suficientemente respondidas, gerando dúvidas quanto ao uso deste tipo de modelo.

Nielsen (1998) apresenta dois métodos para estimar matrizes de viagens a partir de contagens de tráfego. Seus algoritmos foram implementados no TransCAD(C que, segundo Timms (2001) é um dos softwares mais utilizados atualmente. "Este método tem a vantagem dos dados de contagem serem tratados como variáveis es- 
tocásticas, e de poder ser utilizado com qualquer modelo de alocação de tráfego" (Caliper, 2008). Segundo Nielsen (1998), o desenvolvimento destes métodos surgiu de inúmeros casos na Dinamarca, em que houve problemas na atualização de matrizes antigas de má qualidade, utilizando diferentes pacotes de modelagem de tráfego. Assim, o cerne do trabalho de Nielsen (1998) foi a utilidade em aplicações práticas através de métodos de natureza heurística.

O método do caminho único (SPME, do inglês Single Path Matrix Estimation) é de natureza heurística e utiliza apenas as contagens ao longo do caminho ótimo entre cada par OD. O segundo método, de múltiplos caminhos (MPME, do inglês Multiple Path Matrix Estimation) utiliza todas as contagens ao longo de todos os caminhos de acordo com o método das médias sucessivas. Enquanto o foco do primeiro é a facilidade de implementação, o segundo foi desenvolvido para refletir melhor os padrões de escolha de rota.

\section{METODOLOGIA PROPOSTA}

A metodologia proposta é composta por quatro processos: (i) Montagem da rede de transporte em SIG; (ii) Obtenção e tratamento dos dados do SBE; (iii) Aplicação do modelo para determinação da matriz OD semente; (iv) Aplicação do modelo que utiliza contagens volumétricas de passageiros para expandir a matriz OD semente. Para o seu desenvolvimento, são utilizadas três bases de dados primários: (i) banco de dados do SBE; (ii) informações sobre o sistema de transporte para construção da rede em SIG, tais como rede viária, itinerário das linhas do transporte público e zoneamento; (iii) contagens de ocupação das linhas em alguns trechos pré-determinados da rede. O fluxograma da metodologia proposta é apresentado na Erro! Fonte de referência não encontrada. 1 e o detalhamento de cada etapa nas subseções a seguir.

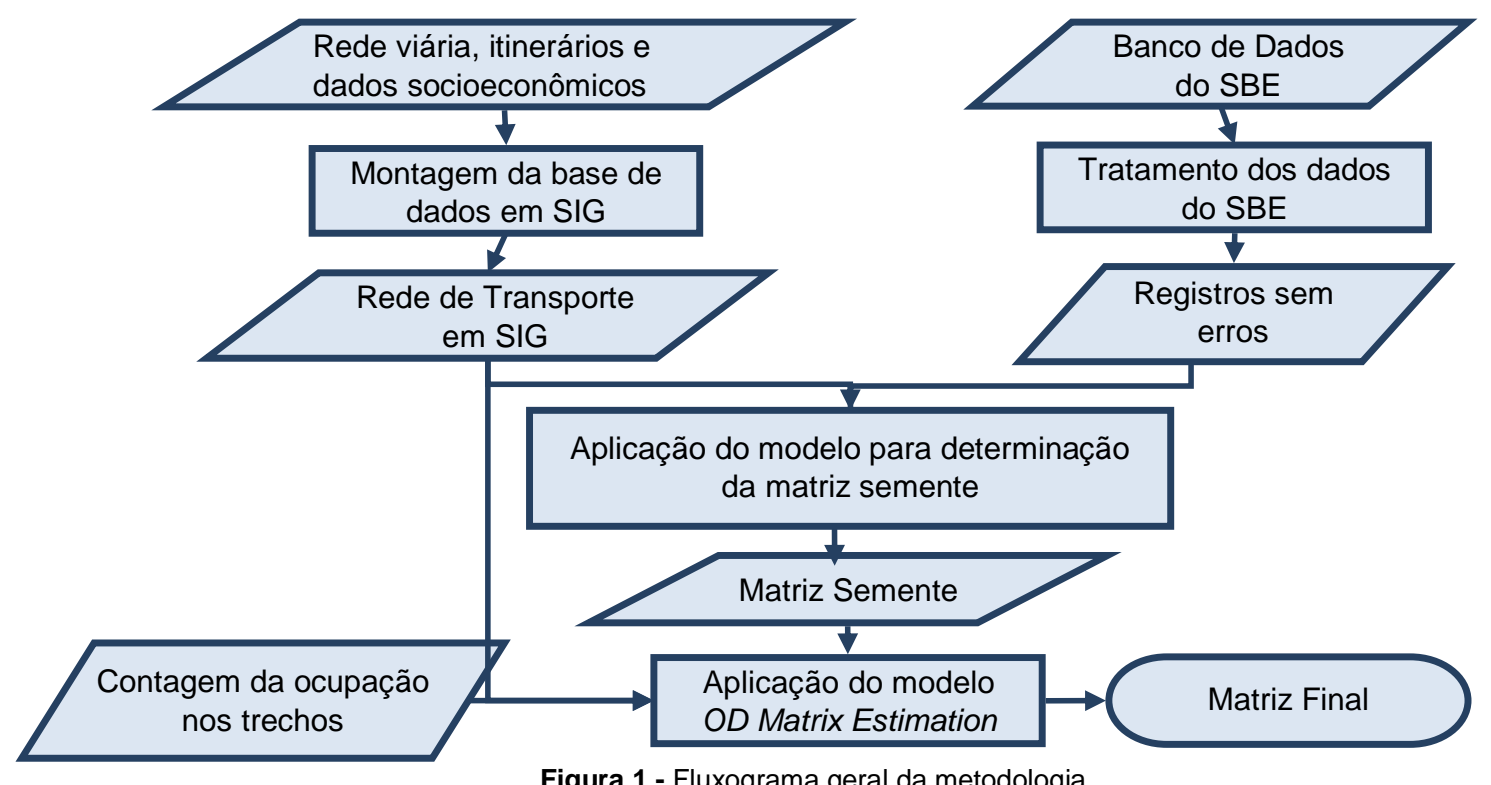

Figura 1 - Fluxograma geral da metodologia

\subsection{Montagem da Rede de Transporte em \\ SIG}

Esta etapa consiste na montagem da base de dados da área de estudo em um software SIG contendo basicamente as seguintes informações: (i) rede viária com informações de distância e velocidade operacional do sistema de transporte; (ii) rede de transporte público; e (iii) divisão da área de estudo em zonas de tráfego.

\subsection{Caracterização e Tratamento dos Dados da Bilhetagem Eletrônica Os SBE podem registrar informações rela-}

tivas às viagens e utilizações do cartão. O primeiro conjunto contempla informações como código de identificação da viagem, número da linha, data e hora do início e término da viagem, total de passageiros pagantes (dinheiro e cartão). $\mathrm{O}$ segundo conjunto de informações refere-se aos registros de todas as transações de tarifa feitas por cartão eletrônico, apresentando informações como código identificador do cartão, código identificador da viagem, data e hora de validação do cartão. 
A operação do SBE depende de interferência humana para registrar o início e término de cada viagem, sendo importante a correta operação por parte do agente de bordo (cobrador). Desta forma, é necessário realizar auditoria nas informações de viagem para eliminar os registros inconsistentes, ou seja, aqueles que apresentam erros de abertura ou fechamento de viagem. Neste trabalho, fez-se necessário assumir que os tempos de viagem de uma linha de ônibus seguem uma distribuição normal, sendo possível estabelecer um intervalo em que estes tempos são considerados válidos. Com relação a uma linha de ônibus, devido às oscilações do tráfego, existe grande variação nos tempos de viagem ao longo de um dia. Portanto, devem-se atribuir curvas específicas para cada período de tempo para uma mesma linha, como faixas horárias. Optou-se por trabalhar a favor da segurança utilizando-se uma probabilidade de $70 \%$ e, assim, eliminando $30 \%$ dos tempos de viagem mais distantes da média.

\subsection{Determinação da Matriz Semente}

O procedimento para determinação da Matriz semente está apresentado na Figura 2 e detalhado a seguir.

A partir da posição do passageiro no momento em que houve o registro do cartão, podese atribuir a zona de tráfego correspondente com a ajuda do banco de dados georreferenciado. A Figura 3 mostra, esquematicamente, uma linha de ônibus que passa por quatro zonas e a extensão do trecho em cada uma delas. Os primeiros $10 \%$ da linha estão dentro da zona 1 , os $30 \%$ seguintes estão na zona 2 , os $40 \%$ seguintes na zona 3 e, por fim, seus $20 \%$ restantes estão dentro da zona 4. No exemplo, observa-se que o passageiro $A$ embarca na zona $1, B$ na zona 2 e o $C$ na zona 3.

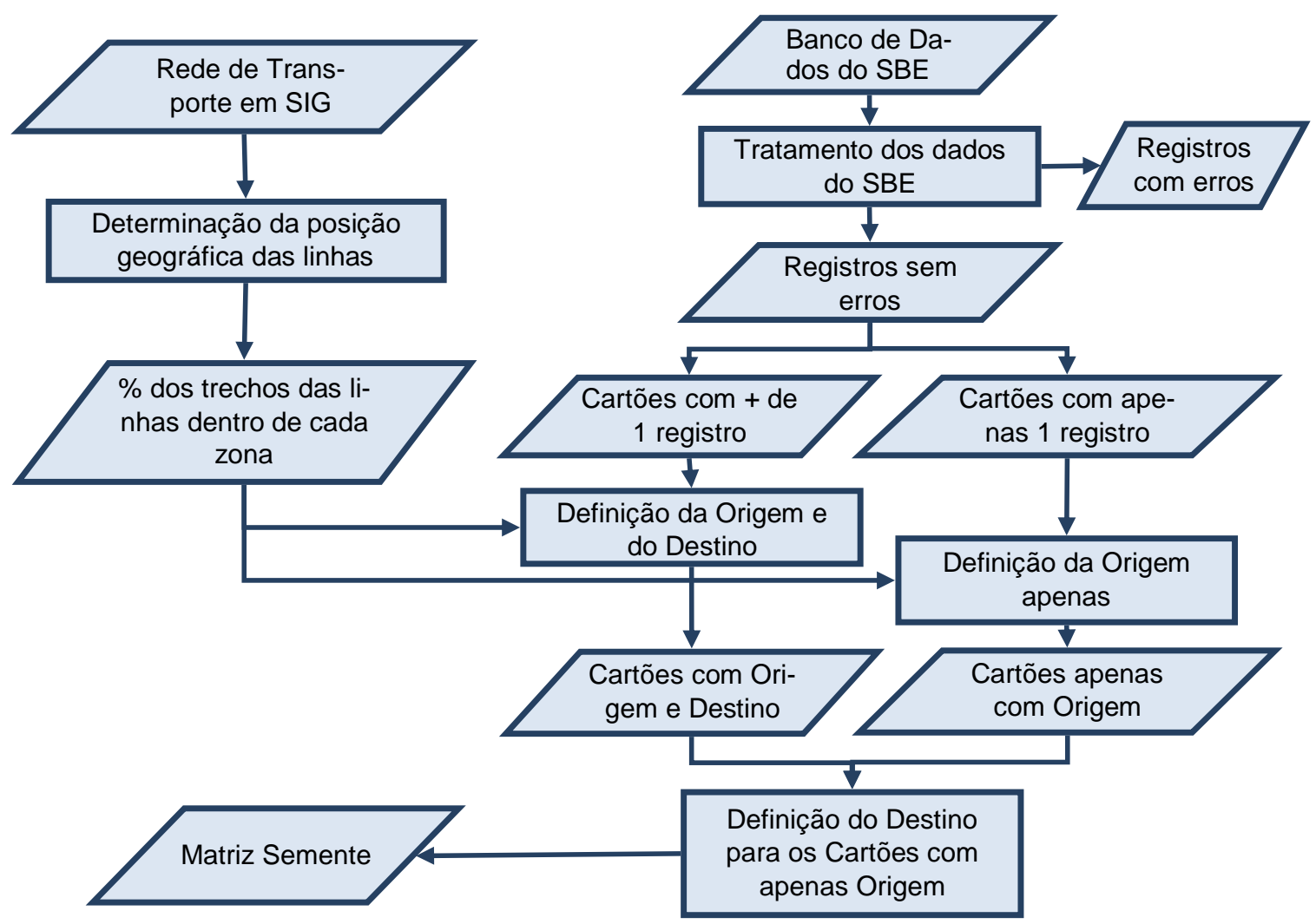

Figura 2 - Fluxograma do procedimento para determinação da matriz semente

\subsubsection{Determinação da Origem}

Inicialmente é definida a posição relativa do passageiro na linha, no momento em que ocorre o pagamento da tarifa. Para isso, é necessário reunir os dois conjuntos de informação do banco de dados do SBE. Como exemplo, considera-se que três passageiros - $A, B$ e $C$ - embarcaram em uma viagem da linha 100 iniciada às 6h20min30s e concluída às 7h30min50s. Cada um deles realizou o pagamento da tarifa em um horário diferente: $A$ às $6 \mathrm{~h} 26 \mathrm{~min} 25 \mathrm{~s}, B$ às $6 \mathrm{~h} 45 \mathrm{~min} 36 \mathrm{~s}$ e $C$ às $7 \mathrm{~h} 10 \mathrm{~min} 32 \mathrm{~s}$, conforme ilustrado na Tabela 1. Considerando como referência os horários de início e término da viagem e de validação do cartão, nota-se que o passageiro 
$A$ validou seu cartão no momento em que o veículo completava $8 \%$ da viagem. Os outros dois,
$B$ e $C$, validaram seus cartões em $36 \%$ e $71 \%$ do itinerário, respectivamente.

Tabela 1 - Definição da posição relativa da entrada do passageiro na linha

\begin{tabular}{lllllll}
\hline Código do cartão & $\begin{array}{l}\text { Hora de registro } \\
\text { do cartão }\end{array}$ & Linha & $\begin{array}{l}\text { Código da } \\
\text { viagem }\end{array}$ & $\begin{array}{l}\text { Início da via- } \\
\text { gem }\end{array}$ & $\begin{array}{l}\text { Final da via- } \\
\text { gem }\end{array}$ & $\begin{array}{l}\text { Percentual } \\
\text { da viagem }\end{array}$ \\
\hline 1029358670 & $6 h 26 m i n 25 s$ & 100 & 203567 & $6 h 20 m i n 30 s$ & $7 h 30 m i n 50 s$ & $8 \%$ \\
1029359326 & $6 h 45 m i n 36 s$ & 100 & 203567 & $6 h 20 m i n 30 s$ & $7 h 30 m i n 50 s$ & $36 \%$ \\
1029405076 & 7h10min32s & 100 & 203567 & $6 h 20 m i n 30 s$ & $7 h 30 m i n 50 s$ & $71 \%$ \\
\hline
\end{tabular}

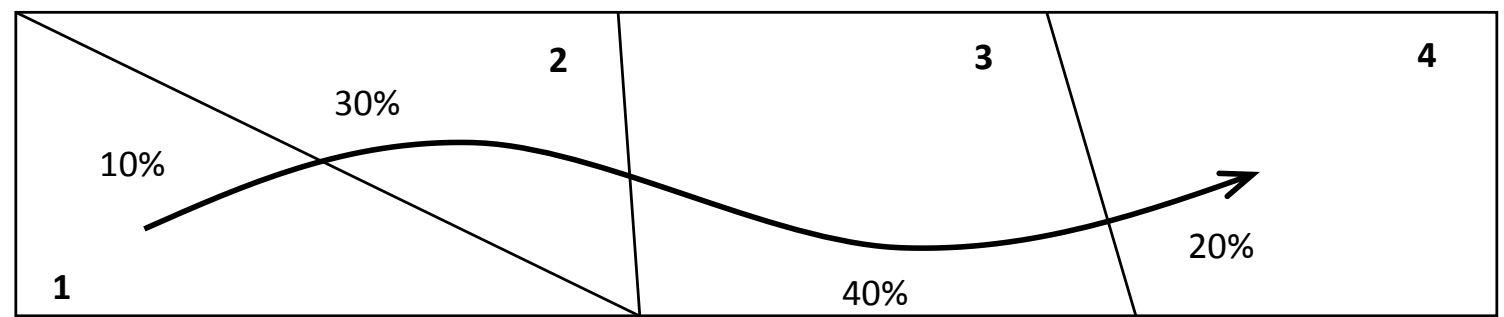

Figura 3 - Localização da linha segundo o zoneamento

A determinação da localização do passageiro no nível de zoneamento minimiza a imprecisão do método causada pela diferença de tempo entre o embarque do passageiro no veículo e a validação do cartão.

\subsubsection{Determinação do Destino}

Depois de definidos os pontos de origem, infere-se sobre o destino dos passageiros. No caso do sistema de transporte público por ônibus, as suposições apresentadas por Zhao (2004) devem ser alteradas com o objetivo de captar de forma mais efetiva os transbordos realizados durante uma viagem. Assim, para os passageiros que precisam utilizar mais de uma linha de ônibus para chegar ao destino final, a viagem ocorre conforme ilustrado na Figura 4: uma pessoa embarca no primeiro ônibus em sua origem, realiza um transbordo e, em seguida, embarca em uma segunda linha para chegar até o ponto de destino. Se fosse considerado o segundo embarque como o destino da primeira linha, teria-se uma situa- ção em que o destino final não é o destino de desejo do passageiro - Figura 4 (b) - mas, o local onde é realizada a troca entre linhas.

Na metodologia proposta adotou-se um intervalo de tempo de 60 minutos a partir da primeira utilização, em que qualquer novo início de viagem é considerado como a efetivação de um transbordo que levará o passageiro ao seu destino final. Por exemplo, se o usuário tem sua primeira utilização do cartão às $6 \mathrm{~h}$, a segunda às $6 \mathrm{~h} 30$ min e a terceira às $12 \mathrm{~h}$, considera-se que a utilização das 6h30min refere-se a um transbordo, já que o intervalo entre esta e a primeira utilização é de apenas 30 minutos. Assim, este registro intermediário é desprezado, e seu destino torna-se o local onde ocorreu a terceira utilização, conforme ilustrado na Figura 4 (c). Adotou-se o intervalo de 60 minutos pois, em muitos sistemas de transporte em que há a bilhetagem eletrônica, é o tempo considerado para conceder descontos na integração temporal.

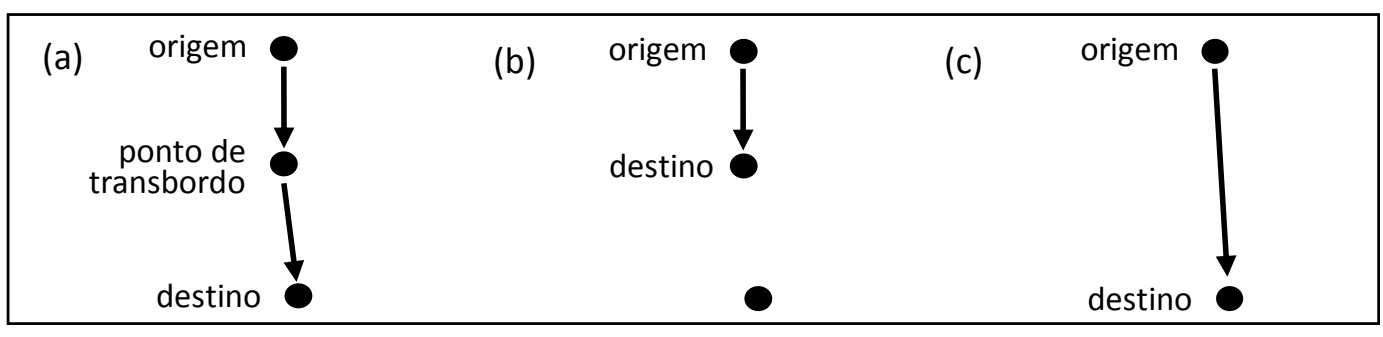

Figura 4 - Situação quando ocorre transbordo

Assim, as suposições para determinar o ponto de destino são as seguintes:

Se o tempo entre a primeira e a segunda utilização do cartão é superior à tolerância de tempo especificada, o destino da primeira viagem ocorre próximo à origem da segunda viagem;

Se o tempo entre a primeira e a segunda 
utilização do cartão é inferior à especificação, o destino da primeira viagem será próximo à origem da terceira viagem.

A base de dados contendo as transações eletrônicas de tarifa pode ser um registro parcial de um deslocamento, nem sempre fidedigno da realidade. De modo geral, a Figura 5 mostra uma situação fictícia considerada para as suposições adotadas nesta metodologia. Em (a), a origem de cada uma das duas viagens representadas foi definida como sendo o local de utilização dos cartões. Em (b) é mostrado como foram deduzidos os locais de destino. Adota-se que o destino da primeira viagem é o local de origem da segunda viagem. Já o destino da segunda viagem, por esta ter sido a última viagem do dia, é o local de origem da primeira viagem.

(a)

(b)

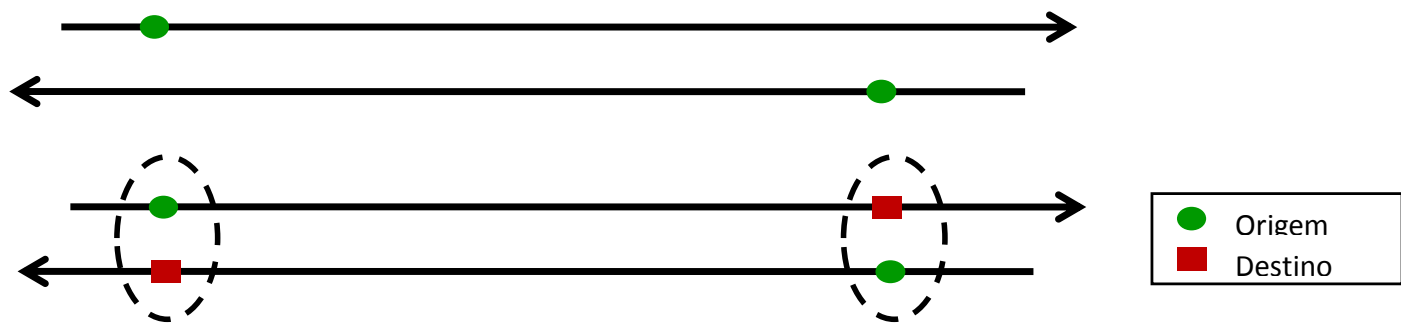

Figura 5 - Definição da origem e inferência do destino

\subsubsection{Tratamento dos Cartões com apenas um} Registro

Para identificar o deslocamento de um passageiro é necessário um mínimo de dois registros de cartão eletrônico. Quando o passageiro realiza apenas uma viagem de transporte público no dia, consegue-se obter apenas a origem de seu deslocamento. Contudo, para o enriquecimento da matriz de transporte, esses registros de passageiros não devem ser desprezados. Neste caso, o tratamento desta informação é motivo de um procedimento adicional.

Utilizando os mesmos conceitos de Farzin (2008), adota-se para determinação dos destinos destas viagens a mesma proporção identificada para as que têm o registro completo. As viagens com destino desconhecido (aquelas com apenas um registro) são alocadas a cada zona de destino

na mesma proporção dos dados completos (com mais de um registro). No exemplo da Tabela 2, a viagem do passageiro ocorreu na linha $51 \mathrm{e}$ teve origem na zona 43. Como ele realizou apenas uma viagem neste dia, a zona de destino não pode ser determinada. Em casos como este, o destino é definido baseando-se no comportamento dos passageiros que também embarcaram na linha 51 , na zona 43 , mas tiveram a identificação completa de seus deslocamentos (origem e destino). Assim, se 50\% deles desembarcam na zona $1,40 \%$, na zona 2 e $10 \%$, na zona 3 , a mesma proporção é atribuída aos passageiros com apenas um registro. $\mathrm{O}$ mesmo tratamento foi realizado com os registros dos passageiros com erro de abertura/fechamento de viagem.

Tabela 2 - Exemplo de registro com erro de abertura/fechamento na segunda utilização

\begin{tabular}{|c|c|c|c|c|c|c|c|c|}
\hline Linha & Viagem & Cartão & Data & Hora & $\begin{array}{l}\text { Hora iní- } \\
\text { cio }\end{array}$ & Hora fim & $\begin{array}{l}\% \text { via- } \\
\text { gem }\end{array}$ & Zona \\
\hline 51 & 14521931 & $\begin{array}{l}231000001080 \\
7\end{array}$ & $16 / 06 / 2010$ & 6h46min22s & 6h30min & 8 h36min & $12,47 \%$ & 43 \\
\hline
\end{tabular}

Ao finalizar esta etapa, obtém-se uma matriz com a informação de origem e destino dos usuários de cartão eletrônico.

\subsection{Procedimento para Estimativa da Matriz Final}

A matriz obtida a partir do banco de dados do SBE (matriz semente) abrange apenas a demanda usuária de cartão eletrônico, não considerando as demais categorias de passageiros.
Nesta etapa será descrito o processo utilizado para extrapolar a matriz semente e incluir todo o universo da demanda usuária do transporte público.

O cálculo da matriz é realizado com a função Transit OD Matrix Estimation (TODME) do software TransCADC, através de modelos matemáticos que consideram contagens de demanda para estimar uma matriz OD. A função TODME utiliza como dados de entrada uma ma- 
triz base (no caso, a matriz semente), as linhas do sistema e contagens de ocupação das linhas em alguns trechos da rede (Nielsen, 1998). A partir destes dados de entrada, o programa realiza a alocação da demanda proveniente da matriz base, gerando fluxos de passageiros. Estes fluxos são comparados com os dados de contagem, e a matriz é modificada individualmente em cada par OD. O resultado é uma Matriz OD que, quando alocada na rede, reproduzirá, de forma consistente, as informações de contagem. O fluxograma da Figura 6 esquematiza o processo.
Segundo Caliper (2008), a matriz base tem duas finalidades: definir as dimensões para a matriz final e fornecer valores iniciais para a matriz estimada. Por isso, é importante que ela seja consistente. Wei et al. (2007 apud Yuanqing et $a l ., 2010)$ afirmam que uma matriz semente de alta qualidade pode melhorar significativamente a precisão da matriz estimada final. Assim, para obter uma matriz inicial com informações coesas, a metodologia empregada utiliza dados sobre as viagens, gerando uma matriz semente consistente que pode garantir bons resultados na estimativa da matriz final.

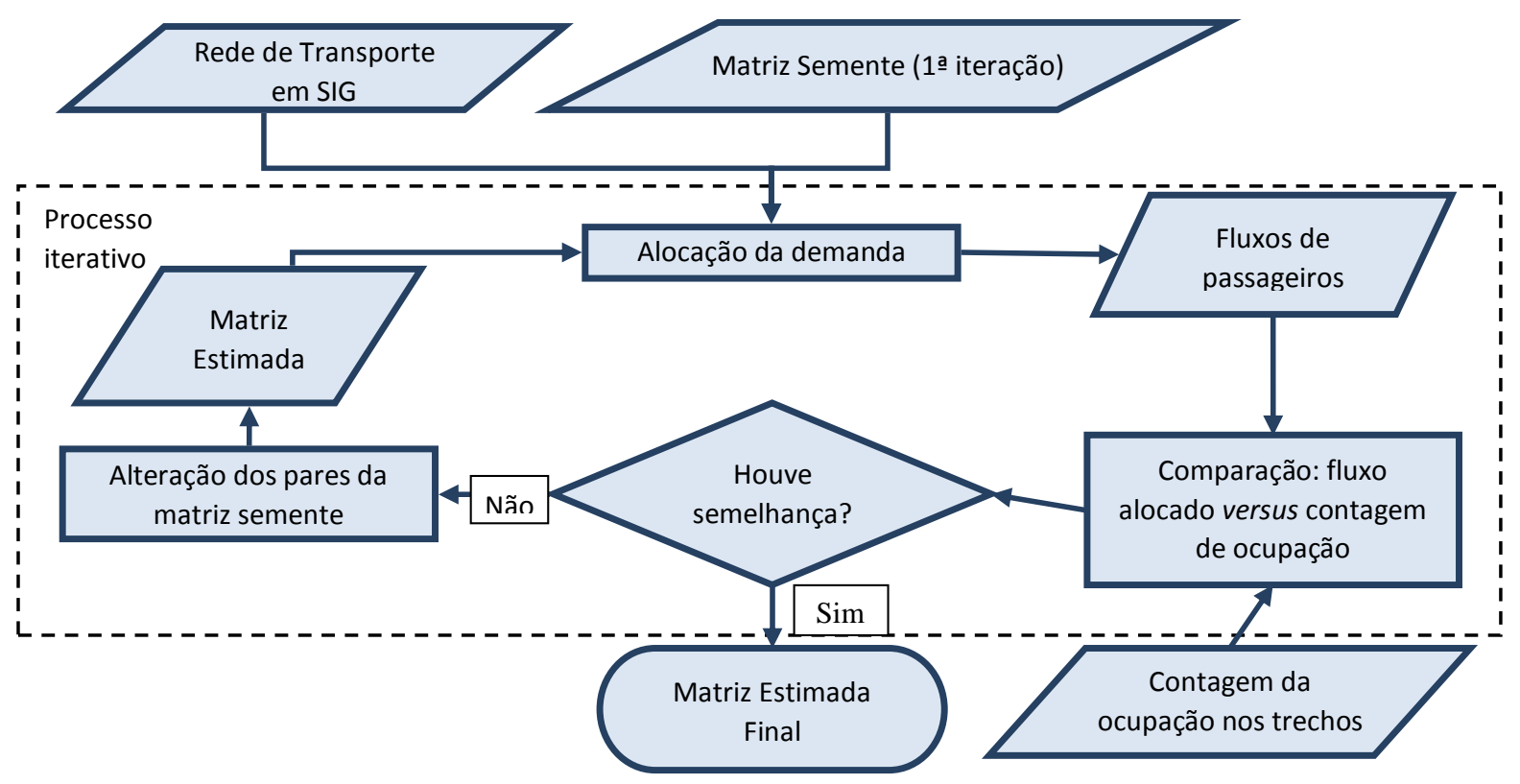

Figura 6 - Fluxograma do procedimento OD Matrix Estimation do TransCADC

\section{APLICAÇÃO DA METODOLOGIA}

Para testar a metodologia proposta, foi realizada uma aplicação ao sistema de transporte público coletivo do município de Maceió, no Estado de Alagoas, cujo SBE está em operação desde 2000.

\subsection{Tratamento da Base de Dados do SBE}

A partir do SBE do transporte municipal de Maceió, dois bancos de dados foram obtidos, um com registros das operações realizadas com o cartão eletrônico e o outro com registros de viagens. A base de dados contempla as informações da semana de 13 a 20 de junho de 2010. No entanto, utilizaram-se os dados do dia 16 de junho, quarta-feira, dia útil típico para testar a metodologia. Neste dia, foram registradas 3.966 viagens, 172.260 validações utilizando 82.614 cartões, além de 125.713 registros de passagei- ros pagantes em dinheiro. O percentual de usuários de cartão eletrônico e passageiros que pagam em dinheiro é de $58 \%$ e $42 \%$, respectivamente.

O passo seguinte consistiu em analisar a consistência dos dados. A principal análise foi avaliar a eficácia do trabalho dos operadores na abertura e no fechamento de viagem, única etapa da operação do sistema de bilhetagem que necessita de interferência humana. Supondo que os tempos de viagens de uma linha de ônibus, dentro de determinado período horário, seguem uma distribuição normal, é possível estabelecer um intervalo em que esses tempos são considerados válidos. Com isso, foram eliminados 784 registros cujos tempos de viagem ficaram fora do intervalo da curva normal, representando aproximadamente $20 \%$ do total. Assim, por estimar a 
matriz OD do pico da manhã, neste estudo foram utilizados apenas os cartões em que, pelo menos, um registro tenha ocorrido entre $5 \mathrm{~h}$ e $7 \mathrm{~h}$, em um total de 51.164 registros com 23.011 cartões, gerando 24.917 embarques no período analisado.

Para os cartões que tiveram apenas um registro na base de dados e aqueles em que um dos registros ocorreu em viagem com erro de abertura e/ou fechamento, foi realizado um tratamento especial. Inicialmente, esses registros foram desconsiderados da base de dados para determinar os locais de origem e destino, sendo posteriormente incorporados à matriz. De um total de 24.917 embarques ocorridos com cartão eletrônico, no horário de pico da manhã, foram utilizados 13.911 para a determinação da matriz OD semente, ou seja, $56 \%$. Considerando que os usuários de cartão representam 58\% do total de usuários do sistema, tem-se que a amostra contempla $32 \%$ do total.

\subsection{Determinação da Matriz Semente}

As bases de dados tratadas foram reunidas para construir a matriz OD. Através da base de dados do SIG, calcula-se o tempo de permanência de uma linha em cada uma das zonas por onde se estende seu itinerário. Com a base de dados do SBE, determina-se o momento em que foram realizadas as transações de cada passageiro usuário de cartão eletrônico. A união destas informações permite definir os locais (zona de tráfego) onde as transações foram efetuadas e, assim, obter a origem e o destino de cada um.

\subsection{Estimativa da Matriz Final}

A estimativa da matriz final consistiu em um procedimento híbrido composto pela expansão da matriz e calibração da rede. $O$ primeiro passo foi a alocação da matriz semente na rede de transporte, sendo utilizado o modelo Pathfinder do TransCAD®. No passo seguinte foram comparados os resultados da alocação com as contagens de volume de passageiros em 28 pontos da rede, cuja localização seguiu critérios como quantidade de linhas no trecho e abrangência do território. No terceiro passo foi aplicado o modelo de determinação de Matriz OD por contagem de fluxo, utilizando a ferramenta do TransCAD(C, OD Matrix Estimation. Ao final do processo, a matriz semente, com 22.085 passageiros, foi expandida resultando em um total de 49.392 passageiros. No quarto passo, o mesmo processo foi aplicado à matriz expandida para alocação na rede de transporte. O quinto passo consistiu no teste de aderência entre a demanda alocada final e a demanda real das linhas. Quando o teste não acusa aderência, a rede é ajustada e todo o processo é repetido. O processo só acaba quando há aderência entre os dois conjuntos de variáveis.

\subsection{Discussão dos Resultados}

A semelhança entre as variáveis observada (demanda real das linhas) e modelada (demanda obtida através da alocação) foi avaliada com o teste de aderência GEH, utilizado em estudos de tráfego e de transportes para comparar dois conjuntos de demanda. Segundo Chaudhry e Ranjitkar (2009), a formulação apresentada na Equação 1 foi desenvolvida para ser tolerante às maiores diferenças em baixos fluxos.

$G E H=\sqrt{\frac{2\left(D_{m}-D_{r}\right)^{2}}{\left(D_{m}+D_{r}\right)}}$

Em que $\mathrm{D}_{\mathrm{m}}=$ demanda modelada e $\mathrm{D}_{\mathrm{r}}=$ demanda real.

Os valores recomendados do GEH são: (i) pelo menos $60 \%$ das linhas devem ter GEH menor que 5; (ii) pelo menos $95 \%$ das linhas devem ter GEH menor que 10, e (iii) todas as linhas devem ter GEH menor que 12.

Os resultados da aplicação foram significativos, havendo aderência entre a demanda total das linhas no modelo $\mathrm{e}$ as respectivas demandas reais, pois para (i) $\mathrm{GEH}<5$ : obtevese $73 \%$, (ii) GEH < 10: obteve-se 95\%, e (iii) GEH $<12$ : obteve-se $100 \%$.

A matriz OD final está representada na Figura 8. Os gráficos do tipo pizza representam o total de passageiros que cada zona atrai e produz, isto é, a proporção entre a produção e a atração. A dimensão do gráfico representa o número de pessoas. As linhas, chamadas de linhas de desejo, indicam a demanda de troca entre duas zonas, sendo que quanto maior a espessura, maior o fluxo de pessoas entre as zonas. Nesta figura estão mostradas apenas as linhas de desejo mais expressivas do sistema em análise.

A comparação dos resultados com o sistema atual é condizente com a realidade, visto que os bairros que mais atraem viagens no período da manhã estão na área central (região sul) e na região turística (área litorânea). $\mathrm{Na}$ área central se concentram estabelecimentos comerciais e de serviço, além de instituições públicas e de ensino. Na região turística estão as princi- 
pais praias, a rede hoteleira e o maior shopping center da cidade. As zonas com maior produção de viagens são situadas na região norte onde es- tão os bairros que concentram unidades residenciais com a presença de grandes conjuntos habitacionais.

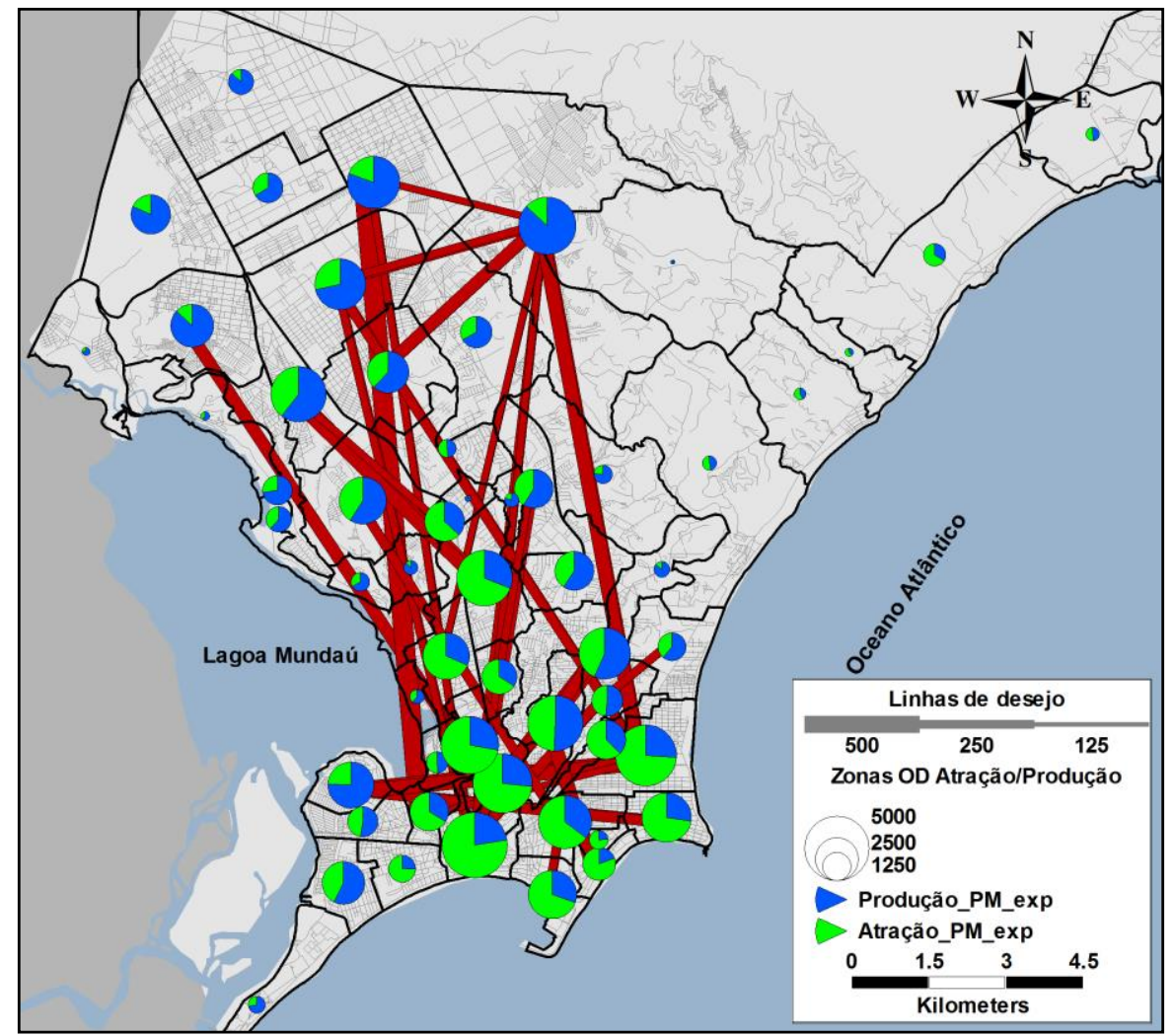

Figura 8 - Representação das zonas de atração e produção e linhas de desejo

\section{CONSIDERAÇÕES FINAIS}

Os dados do Sistema de Bilhetagem Eletrônica fornecem uma infinidade de informações importantes para os estudos relativos ao transporte público, sendo uma fonte de dados que pode ser utilizada na determinação de uma matriz OD de passageiros. Assim, neste trabalho foi apresentada uma metodologia para obtenção de uma matriz OD de passageiros do transporte público por ônibus utilizando os dados de um SBE. $O$ procedimento inicia-se com a determinação de uma matriz semente amostral que contempla apenas os passageiros usuários de cartão eletrônico. Em seguida esta matriz é expandida através de um processo que utiliza contagens de fluxos de passageiros. O processo para expansão da matriz semente se baseia em contagens, que determina o número total de passageiros incluindo aqueles que não são contabilizados pelo SBE. Desta forma, os deslocamentos, representados pela matriz final, não ficam condicionados ao comportamento típico dos usuários de cartão eletrônico.
A aplicação da metodologia indica que esta é simples, conveniente e prática, podendo ser utilizada para estudos relacionados a transporte público. Para a aplicação da metodologia são necessários dados do SBE, de contagem dos fluxos de passageiros em trechos distintos da rede e a rede de transporte em SIG. Como não foram realizadas pesquisas de campo para a aplicação da metodologia, os fluxos de passageiros foram determinados através de relações matemáticas utilizando dados de demanda real das linhas de ônibus e dados internos ao modelo.

As limitações deste trabalho estão relacionadas (i) à simplificação da análise estatística feita sobre os dados do SBE, (ii) à falta de um processo para medir com exatidão a qualidade da matriz final, e (iii) ao pressuposto de que todos os deslocamentos diários dos passageiros são realizados pelo modo ônibus, assumido para determinar a origem e o destino dos deslocamentos. Conforme mencionado anteriormente, a imprecisão causada pela diferença entre o tempo 
de embarque do passageiro e a validação do cartão é minimizada por se considerar a localização (da origem e do destino das viagens) no nível do zoneamento. Tratando-se desta diferença, podem existir casos em que o passageiro deixa para validar seu cartão no momento do desembarque, entretanto, esse impacto é pouco significativo já que há uma limitação de espaço físico nos veículos entre a porta de entrada e a catraca onde ocorre a validação. Além disso, na fase de determinação da matriz final, em que os fluxos são ajustados pelas contagens de passageiros, as possíveis imprecisões podem ser corrigidas. Além disso, ressalta-se que a aplicação da metodologia refere-se apenas à rede de transporte público, não configurando, necessariamente, origem e destino da viagem em si. Ademais, em aplicações futuras da metodologia, é necessário considerar os tempos de integração temporal das cidades em estudo, que deve ser compatível com o porte da rede de transporte da localidade. Outro ponto a considerar são os casos em que ocorre integração modal no sistema.

Apesar das limitações, os resultados indicam a aplicabilidade da metodologia para obtenção de dados de matriz origem destino do transporte público por ônibus, com elevada frequência, visto que após construído o procedimento computacional para a obtenção da referida matriz, é possível, facilmente, atualizála com os novos dados do Sistema de Bilhetagem Eletrônica. Neste contexto, os planejadores urbanos terão sempre informações atualizadas para a tomada de decisão, a custo baixo.

\section{AGRADECIMENTOS}

Os autores agradecem à CAPES, CNPQ e FAPEMIG pelo suporte no desenvolvimento da pesquisa.

\section{REFERÊNCIAS}

ANTP (1997) Transporte Humano: Cidades com Qualidade de Vida. Associação Nacional de Transporte Público. São Paulo.

ABRAHAMSSON, T. (1998) Estimation of Origin-Destination Matrices Using Traffic Counts - A Literature Survey. International Institute for Applied Systems Analysis, Luxemburg.

BERTONCINI, B. V.; LOUREIRO C. F. G.; KAWAMOTO E. (2013) Proposta e avaliação de algoritmo de médias sucessivas para reconstrução da matriz origem-destino sintética. $\begin{array}{lllll}\text { Transportes, } & \text { v. } 21, \quad \text { n. 21-29. } & \text { DOI: }\end{array}$ $10.4237 /$ transportes.v21i2.697
BERTONCINI, B. V. (2007) Uma proposta de carregamento incremental de fluxos veiculares para estimação de matrix $O-D$ sintética. Escola de Engenharia de São Carlos da Universidade de São Paulo, São Carlos.

CALIPER (2008) TransCAD Transportation Planning Software - Travel Demand Modeling with TransCAD 5.0 - User's Guide. Caliper Corporation, Newton.

CASCETTA E. (1984) Estimation of Trip Matrices from Traffic Counts and Survey Data: A Generalised Least Squares Estimator. Transportation Research Part B, v. 18, n. 4-5, p. 289299. DOI: 10.1016/0191-2615(84)90012-2

CHAPLEAU, R.; TRÉPANIER, M.; CHU, K. K. (2008) The Ultimate Survey for Transit Planning: Complete Information with Smart Card Data and GIS. 8th International Conference on Survey Methods in Transport: Harmonization and Data Comparability, Annecy.

CHAUDHRY, M. S.; RANJITKAR, P. (2009) Capacity Analysis of Signalised Intersection using Micro-simulation. 32nd Australian Transport Research Forum, Auckland.

CUI, A. (2006) Bus Passenger Origin-Destination Matrix Estimation Using Automated Data Collection Systems. Thesis. Massachusetts Institute of Technology, Boston.

FARZIN, J. M. (2008) Constructing an Automated Bus OriginDestination Matrix Using Farecard and Global Positioning System Data in São Paulo, Brazil. Transportation Research Record: Journal of the Transportation Research Board, Washington, n. 2072, p. 30-37. DOI: 10.3141/2072-04.

GORDILLO, F. (2006) The Value of Automated Fare Collection Data for Transit Planning: An Example of Rail Transit OD Matrix Estimation. Thesis. Massachusetts Institute of Technology, Boston.

LIANFU, Z.; SHUZHI, Z.; YONGGANG Z.; ZIYIN, Z. (2007) Study on the Method of Constructing Bus Stops OD Matrix Based on IC Card Data. International Conference on Wireless Communications, Networking, and Mobile Computing, Shanghai. P. 3147-3150. DOI: 10.1109/WICOM.2007.780

MUNIZAGA, M.; PALMA, C. (2012) Estimation of a Disaggregate Multimodal Public Transport OD Matrix from Passive Smart Card Data from Santiago, Chile. Transportation Research Part C: Emerging Technologies, v. 24, p. 9-18. DOI: 10.1016/j.trc.2012.01.007

MUNIZAGA, M.; PALMA, C.; MORA, P. (2010) Public Transport OD Matrix Estimation from Smart Card Payment System Data. Proceedings from 12th World Conference on Transport Research, Lisboa.

NIELSEN, O. A. (1998) Two New Methods for Estimating Trip Matrices from Traffic Counts. In: ORTÚZAR, J. D. D.; HENSHER, D. A.; JARA-DIAZ, S. Travel Behaviour Research: Updating the State of Play. Oxford: Elsevier Science Ltd. Cap. 13, p. $221-250$

NTU (2005) Novas Tendências em Política Tarifária. Associação Nacional das Empresas de Transportes Urbanos, Brasília.

NTU (2013) Vale-transporte 2012. Associação Nacional das Empresas de Transportes Urbanos, Brasília.

TIMMS, P. (2001) A philosophical context for methods to estimate origin-destination trip matrices using link counts. Transport Reviews, v. 21, n. 3, p. 269-301. DOI: $10.1080 / 713868144$ 
TRÉPANIER, M.; MORENCY, C.; BLANCHETTE, C. (2008) Les systèmes de paiement par cartes à puces: un complément aux enquêtes origine-destination? 43e congrès de l'Association québécoise du transport et des routes, Quebec.

YUANQING, W., LIU, Y., YIN, G., MENGMENG, Z. (2010) OD Matrix Estimation for Urban Expressway. Journal of Transportation Systems Engineering and Information Technology, n. 10, p. 83-87. DOI: 10.1016/S15706672(09)60035-0

WEI X. L., LI, H. F., GENG Y. B. (2007) Contrast research on
OD matrix estimation result with TransCAD software. Highway, 6: $122-128$.

WILLUMSEN, L.G. (1981) Simplified transport models based on traffic counts. Transportation, n. 10, p. 257-278. DOI: 10.1007/BF00148462.

ZHAO, J. (2004) The Planning and Analysis Implications of Automated Data Collection System: Rail Transit OD Matrix Inference and Path Choice Modeling Examples. Thesis. Massachusetts Institute of Technology, Boston. 